\title{
Joint Channel Decoding and Physical-Layer Network Coding in Two-Way QPSK Relay Systems by a Generalized Sum-Product Algorithm
}

\author{
Dirk Wübben \\ Department of Communications Engineering, University of Bremen, Otto-Hahn-Allee, 28359 Bremen, Germany \\ Phone: +4942121862385 \\ wuebbendant.uni-bremen. de
}

\begin{abstract}
A physical-layer network coded two-way relay system applying Low-Density Parity-Check (LDPC) codes for error correction is considered in this paper, where two sources A and $B$ desire to exchange information with each other by the help of a relay $R$. The critical process in such a system is the calculation of the network-coded transmit word at the relay on basis of the superimposed channel-coded QPSK words of the two sources. For this joint channel-decoding and network-encoding task a generalized Sum-Product Algorithm (SPA) over $\mathbb{F}_{16}$ is developed. This novel iterative decoding approach outperforms other recently proposed schemes as demonstrated by simulations.
\end{abstract}

\section{INTRODUCTION}

The concept of network coding enables throughput enhancements by allowing the intermediate nodes to perform operations on the incoming data [1]. The basic idea can be applied to wireless two-way relay systems, where two sources $\mathrm{A}$ and $\mathrm{B}$ want to exchange information with each other by the help of a relay node $\mathrm{R}$. In a direct approach the sources send their information one after the other to $\mathrm{R}$ and the relay transmits the XOR of both received messages to the sources in a third time slot exploring the broadcast nature of the wireless channel [2]. As both sources know what they have transmitted previously, they can estimate the message of the other source by simple XOR operation. Allowing A and B to transmit their information simultaneously to $\mathrm{R}$, the number of time slots for information exchange can be reduced to two. As the information of both sources are combined during the transmission, this scheme is called physical-layer network coding (PLNC) [3]. In PLNC a relay is not required to decode the information of the two sources explicitly, but it can map the received signal directly to a network encoded signal to be relayed. Such modulation-demodulation approaches neglecting the impact of channel coding have been considered in [3], [4]. Extensions for Joint Channel decoding and physicallayer Network Coding (JCNC) have been introduced in [5], [6]. Applying the same linear channel code at both source nodes, the XOR of both source codewords is also a valid codeword. Thus, the received signal can be decoded to the XOR of the source information at the relay without changing

This work was supported in part by the German Research Foundation (DFG) under grant Wu 499/7. the decoding algorithm. As this approach looses information, decoding based on the Arithmetic-Sum (AS-JCNC) of the source codewords was proposed for Repeat Accumulate (RA) codes in [7] and for LDPC codes in [8], [9] requiring an adapted channel decoder at the relay. As these schemes were restricted to AWGN channels with BPSK transmission, a generalization with respect to fading channels was presented in [10]. In this contribution, we extend the proposed scheme to QPSK transmission, leading to sixteen different undisturbed receive values. For this system we develop a Generalized SumProduct Algorithm (G-SPA) over the Galois field $\mathbb{F}_{16}$ [11], [12] resulting in improved performance.

The remainder of this paper is organized as follows. In Section II the two-way relaying system is introduced. In Section III common schemes for joint channel decoding and physical-layer network coding from the literature are reviewed. Our new approach is developed in Section IV and the performance of the different schemes is compared in Section V. The paper is finished by a summary in Section VI.

\section{SySTEM MODEL}

The two-way relay system with two sources $\mathrm{A}$ and $\mathrm{B}$, and one relay $\mathrm{R}$ is shown in Fig. 1. Both sources wish to exchange information with each other by the help of $\mathrm{R}$ as no direct link is present. It is assumed, that all nodes are perfectly synchronized and operate in half-duplex mode, i.e., they can not receive and transmit simultaneously.

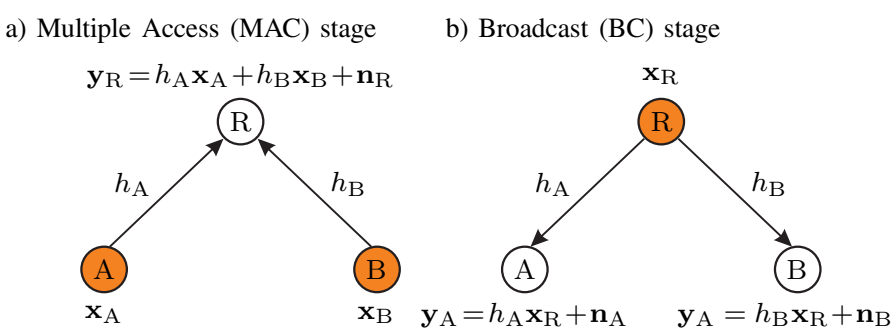

Fig. 1. Two source A and B exchange information with each other by the relay $\mathrm{R}$. The communication consists of MAC and a BC stage.

The binary information words of $\mathrm{A}$ and $\mathrm{B}$ of length $K$ are denoted by $\mathbf{b}_{\mathrm{A}}$ and $\mathbf{b}_{\mathrm{B}}$, respectively. Both sources encode their information by the same linear channel code $\Gamma$ with code rate $R_{c}=K / N$ into the codewords $\mathbf{c}_{\mathrm{A}}=\Gamma\left(\mathbf{b}_{\mathrm{A}}\right)$ and $\mathbf{c}_{\mathrm{B}}=\Gamma\left(\mathbf{b}_{\mathrm{B}}\right)$, 
denoted as source codewords. Afterwards, the codewords are QPSK-modulated to $\mathbf{x}_{\mathrm{A}}=\mathcal{M}\left\{\mathbf{c}_{\mathrm{A}}\right\}$ and $\mathbf{x}_{\mathrm{B}}=\mathcal{M}\left\{\mathbf{c}_{\mathrm{B}}\right\}$ according to the mapping rule $\{00,10,01,11\} \rightarrow\{1, j,-1,-j\}$. The transmit word $\mathbf{x}_{\mathrm{A}}=\left[\begin{array}{llll}x_{\mathrm{A}}(1) & \ldots & x_{\mathrm{A}}(L)\end{array}\right]$ of source $\mathrm{A}$ consists of $L=N / 2$ symbols, where the symbol $x_{\mathrm{A}}(\ell)=$ $\mathcal{M}\left\{c_{\mathrm{A}}(\ell)\right\}$ depends on the bit tuple $c_{\mathrm{A}}(\ell)=\left[c_{\mathrm{A}, 1}(\ell) c_{\mathrm{A}, 2}(\ell)\right]$. In order to ease the notation, the time index $\ell$ will be avoided whenever possible, e.g., $c_{\mathrm{A}}$ denotes an arbitrary tuple of the source codeword $\mathbf{c}_{\mathrm{A}}$. The same argument holds for the elements of the remaining vectors.

The two-way relaying transmission consists of a multiple access (MAC) and a broadcast (BC) stage. In the MAC stage, both sources transmit $\mathrm{x}_{\mathrm{A}}$ and $\mathrm{x}_{\mathrm{B}}$ to the relay $\mathrm{R}$ simultaneously. Thus, the $\ell$-th received signal at $\mathrm{R}$ is given by the linear superposition of the transmit signals weighted by the fading coefficients $h_{\mathrm{A}}(\ell)$ and $h_{\mathrm{B}}(\ell)$, i.e.,

$$
y_{\mathrm{R}}(\ell)=h_{\mathrm{A}}(\ell) x_{\mathrm{A}}(\ell)+h_{\mathrm{B}}(\ell) x_{\mathrm{B}}(\ell)+n_{\mathrm{R}}(\ell) .
$$

The elements of the noise vector $\mathbf{n}_{R}$ at $R$ are i.i.d zeromean complex Gaussian random variables with variance $\sigma_{n}^{2}$. For network coding the relay performs an estimation with respect to the XOR of the source codewords $\mathbf{c}_{\mathrm{A} \oplus \mathrm{B}}=\mathbf{c}_{\mathrm{A}} \oplus \mathbf{c}_{\mathrm{B}}$ based on $\mathbf{y}_{R}$. This estimated relay codeword $\mathbf{c}_{\mathrm{R}}=\hat{\mathbf{c}}_{\mathrm{A} \oplus \mathrm{B}}$ is again QPSK-modulated and the relay transmits $\mathbf{x}_{\mathrm{R}}=\mathcal{M}\left\{\mathbf{c}_{\mathrm{R}}\right\}$ towards both sources $\mathrm{A}$ and $\mathrm{B}$ in the $\mathrm{BC}$ stage. For simplicity it is assumed, that the transmission channels are reciprocal, the relay $\mathrm{R}$ transmits with the same power as both sources, and the noise variance is again $\sigma_{n}^{2}$. Thus, the $\ell$-th received signals at $\mathrm{A}$ and $\mathrm{B}$ are given by $y_{\mathrm{A}}(\ell)=h_{\mathrm{A}}(\ell) x_{\mathrm{R}}(\ell)+n_{\mathrm{A}}(\ell)$ and $y_{\mathrm{B}}(\ell)=h_{\mathrm{B}}(\ell) x_{\mathrm{R}}(\ell)+n_{\mathrm{B}}(\ell)$. Both sources $\mathrm{A}$ and $\mathrm{B}$ can then estimate the information $\hat{\mathbf{b}}_{\mathrm{R}, \mathrm{A}}$ and $\hat{\mathbf{b}}_{\mathrm{R}, \mathrm{B}}$ from $\mathrm{y}_{\mathrm{A}}$ and $\mathbf{y}_{\mathrm{B}}$, respectively. Since both sources know what they have transmitted in the MAC stage, the information from the other source can be obtained by simple XOR, i.e., $\hat{\mathbf{b}}_{\mathrm{B}}=\hat{\mathbf{b}}_{\mathrm{R}, \mathrm{A}} \oplus \mathbf{b}_{\mathrm{A}}$ and $\hat{\mathbf{b}}_{\mathrm{A}}=\hat{\mathbf{b}}_{\mathrm{R}, \mathrm{B}} \oplus \mathbf{b}_{\mathrm{B}}$. For the critical step of channel decoding and physical-layer network encoding at the relay, a new decoding algorithm for $\mathbf{y}_{\mathrm{R}} \rightarrow \mathbf{c}_{\mathrm{R}}$ is derived in this paper for QPSK transmission. This new algorithm makes full use of both channel codes in combination with the PLNC scheme.

\begin{tabular}{|c||c|c||c|c||c|c||c|}
\hline$i$ & $c_{\mathrm{A}}$ & $c_{\mathrm{B}}$ & $c_{\mathrm{A} \oplus \mathrm{B}}$ & $c_{\mathrm{AB}}$ & $x_{\mathrm{A}}$ & $x_{\mathrm{B}}$ & $s_{\mathrm{AB}}$ \\
\hline 0 & 00 & 00 & 00 & 0 & 1 & 1 & $h_{\mathrm{A}}+h_{\mathrm{B}}$ \\
1 & 10 & 00 & 10 & 1 & $j$ & 1 & $j h_{\mathrm{A}}+h_{\mathrm{B}}$ \\
2 & 01 & 00 & 01 & $D$ & -1 & 1 & $-h_{\mathrm{A}}+h_{\mathrm{B}}$ \\
3 & 11 & 00 & 11 & $1+D$ & $-j$ & 1 & $j h_{\mathrm{A}}+h_{\mathrm{B}}$ \\
4 & 00 & 10 & 10 & $D^{2}$ & 1 & $j$ & $h_{\mathrm{A}}+h_{\mathrm{B}}$ \\
5 & 10 & 10 & 00 & $1+D^{2}$ & $j$ & $j$ & $j h_{\mathrm{A}}+j h_{\mathrm{B}}$ \\
6 & 01 & 10 & 11 & $D+D^{2}$ & -1 & $j$ & $-h_{\mathrm{A}}+j h_{\mathrm{B}}$ \\
7 & 11 & 10 & 01 & $1+D+D^{2}$ & $-j$ & $j$ & $-j h_{\mathrm{A}}+j h_{\mathrm{B}}$ \\
8 & 00 & 01 & 01 & $D^{3}$ & 1 & -1 & $h_{\mathrm{A}}-h_{\mathrm{B}}$ \\
9 & 10 & 01 & 11 & $1+D^{3}$ & $j$ & -1 & $j h_{\mathrm{A}}-h_{\mathrm{B}}$ \\
10 & 01 & 01 & 00 & $D+D^{3}$ & -1 & -1 & $-h_{\mathrm{A}}-h_{\mathrm{B}}$ \\
11 & 11 & 01 & 10 & $1+D+D^{3}$ & $-j$ & -1 & $-j h_{\mathrm{A}}-h_{\mathrm{B}}$ \\
12 & 00 & 11 & 11 & $D^{2}+D^{3}$ & 1 & $-j$ & $h_{\mathrm{A}}-j h_{\mathrm{B}}$ \\
13 & 10 & 11 & 01 & $1+D^{2}+D^{3}$ & $j$ & $-j$ & $j h_{\mathrm{A}}-j h_{\mathrm{B}}$ \\
14 & 01 & 11 & 10 & $D+D^{2}+D^{3}$ & -1 & $-j$ & $-h_{\mathrm{A}}-j h_{\mathrm{B}}$ \\
15 & 11 & 11 & 00 & $1+D+D^{2}+D^{3}$ & $-j$ & $-j$ & $-j h_{\mathrm{A}}-j h_{\mathrm{B}}$ \\
\hline
\end{tabular}

TABLE I

Mapping rules for code bit tuples $\left(c_{\mathrm{A}}, c_{\mathrm{B}}\right)$ and transmit signals $\left(x_{\mathrm{A}}, x_{\mathrm{B}}\right)$.
Subsequently, some basic relations between the different occurring signals and their probabilities are given. Tab. I summarizes the basic relationships between the occurring code bit tuples $c_{\mathrm{A}}=\left[c_{\mathrm{A}, 1} c_{\mathrm{A}, 2}\right], c_{\mathrm{B}}=\left[c_{\mathrm{B}, 1} c_{\mathrm{B}, 2}\right]$ and the corresponding QPSK signals $x_{\mathrm{A}}, x_{\mathrm{B}}$. For the later derivations we also include the XOR $c_{\mathrm{A} \oplus \mathrm{B}}=c_{\mathrm{A}} \oplus c_{\mathrm{B}}=\left[c_{\mathrm{A}, 1} \oplus c_{\mathrm{B}, 1} c_{\mathrm{A}, 2} \oplus c_{\mathrm{B}, 2}\right]$ of the code symbols and the sixteen different noise-free signal levels at the receiver side $s_{\mathrm{AB}} \in \mathcal{S}_{\mathrm{AB}}$ following (1). Furthermore, the 16-ary symbol $c_{\mathrm{AB}}=c_{\mathrm{A}, 1}+c_{\mathrm{A}, 2} D+c_{\mathrm{B}, 1} D^{2}+c_{\mathrm{B}, 2} D^{3} \triangleq$ $\left[c_{\mathrm{A}, 1} c_{\mathrm{A}, 2} c_{\mathrm{B}, 1} c_{\mathrm{B}, 2}\right]$ is defined as a short hand notation for the 16 different combinations of $c_{\mathrm{A}}$ and $c_{\mathrm{B}}$, i.e, $c_{\mathrm{AB}} \in \mathcal{C}_{\mathrm{AB}}$ with Galois Field $\mathcal{C}_{\mathrm{AB}}=\mathbb{F}_{16}$ (in polynomial description with indeterminate $D$ ). For notational convenience, we will make use of the polynomial presentation as well as the 4-tuple representation for $c_{\mathrm{AB}}$. Thus, $c_{\mathrm{AB}}=\mathcal{C}_{\mathrm{AB}}(i)$ and $s_{\mathrm{AB}}=\mathcal{S}_{\mathrm{AB}}(i)$ represent the $i$-th event $(0 \leq i \leq 15)$ in $\mathbb{F}_{16}$ and in the receive signal space, respectively.

The a-priori probabilities for $c_{\mathrm{AB}}=\mathcal{C}_{\mathrm{AB}}(i)$ and $s_{\mathrm{AB}}=$ $\mathcal{S}_{\mathrm{AB}}(i)$ are given for equally likely code symbols by

$$
\operatorname{Pr}\left\{c_{\mathrm{AB}}=\mathcal{C}_{\mathrm{AB}}(i)\right\}=\operatorname{Pr}\left\{s_{\mathrm{AB}}=\mathcal{S}_{\mathrm{AB}}(i)\right\}=\frac{1}{16} .
$$

The probability density for $y_{\mathrm{R}}$ given the noise-free receive signal $s_{\mathrm{AB}} \in \mathcal{S}_{\mathrm{AB}}$ corresponds to

$$
p\left\{y_{\mathrm{R}} \mid s_{\mathrm{AB}}=\mathcal{S}_{\mathrm{AB}}(i)\right\}=\frac{1}{\pi \sigma_{n}^{2}} \exp \left(-\frac{\left|y_{\mathrm{R}}-\mathcal{S}_{\mathrm{AB}}(i)\right|^{2}}{x \sigma_{n}^{2}}\right) .
$$

Thus, the probability that the signal $s_{\mathrm{AB}}=\mathcal{S}_{\mathrm{AB}}(i)$ was transmitted given the current receive signal $y_{\mathrm{R}}$ is

$$
\begin{aligned}
P_{i} & =\operatorname{Pr}\left\{c_{\mathrm{AB}}=\mathcal{C}_{\mathrm{AB}}(i) \mid y_{\mathrm{R}}\right\}=\operatorname{Pr}\left\{s_{\mathrm{AB}}=\mathcal{S}_{\mathrm{AB}}(i) \mid y_{\mathrm{R}}\right\} \\
& =p\left\{y_{\mathrm{R}} \mid s_{\mathrm{AB}}=\mathcal{S}_{\mathrm{AB}}(i)\right\} \frac{\operatorname{Pr}\left\{s_{\mathrm{AB}}=\mathcal{S}_{\mathrm{AB}}(i)\right\}}{\operatorname{Pr}\left\{y_{\mathrm{R}}\right\}} \\
& =p\left\{y_{\mathrm{R}} \mid s_{\mathrm{AB}}=\mathcal{S}_{\mathrm{AB}}(i)\right\} \frac{1}{C} .
\end{aligned}
$$

As the sum over all probabilities $P_{i}$ equals 1 , the constant $C=$ $16 \operatorname{Pr}\left\{y_{\mathrm{R}}\right\}$ in (4) can be calculated and is used to normalize the probabilities $P_{i}$.

\section{Common Decoding Schemes}

In this section two common approaches to perform the decoding at the relay are shortly repeated.

\section{A. Separated Channel Decoding (SCD)}

The estimation of the source information at the relay can be interpreted as the traditional multiple access problem, which aims to estimate $\mathbf{c}_{\mathrm{A}}$ and $\mathbf{c}_{\mathrm{B}}$ explicitly by Separated Channel Decoding (SCD). One simple approach performs a decoding of $\mathbf{c}_{\mathrm{A}}$ and $\mathbf{c}_{\mathrm{B}}$ on basis of the receive word $\mathbf{y}_{\mathrm{R}}$ in parallel (called P-SCD). The a-posteriori probabilities (APPs) for $c_{\mathrm{A}}$ given the receive signal $y_{\mathrm{R}}$ are given by

$$
\operatorname{Pr}\left\{c_{\mathrm{A}, \nu}=\xi \mid y_{\mathrm{R}}\right\}=\sum_{i \in \Omega_{\mathrm{A}, \nu}^{\xi}} P_{i}
$$

and can be calculated using (4). Here, $\Omega_{\mathrm{A}, \nu}^{\xi}$ defines the set of indices, where bit $c_{\mathrm{A}, \nu}$ is equal to $\xi=\{0,1\}$ according to 
Tab. I. For example, $\Omega_{\mathrm{A}, 1}^{0}=\{0,2,4,6,8,10,12,14\}$ indicates all events where $c_{\mathrm{A}, 1}=0$. For decoding, the APP vector $\left[\operatorname{Pr}\left\{c_{\mathrm{A}, \nu}=0 \mid y_{\mathrm{R}}\right\} \quad \operatorname{Pr}\left\{c_{\mathrm{A}, \nu}=1 \mid y_{\mathrm{R}}\right\}\right]$ or the corresponding Log-Likelihood Ratio (LLR)

$$
\lambda_{\mathrm{A}, \nu}=\ln \left(\frac{\operatorname{Pr}\left\{c_{\mathrm{A}, \nu}=0 \mid y_{\mathrm{R}}\right\}}{\operatorname{Pr}\left\{c_{\mathrm{A}, \nu}=1 \mid y_{\mathrm{R}}\right\}}\right)
$$

is fed to the Sum-Product Algorithm (SPA) for each bit of the code word. At the output the estimate $\hat{\mathbf{c}}_{\mathrm{A}}$ for the codeword transmitted by source A is achieved. Similarly, the APPs for $c_{\mathrm{B}, \nu}$ are calculated and the APP vector $\left[\operatorname{Pr}\left\{c_{\mathrm{B}, \nu}=0 \mid y_{\mathrm{R}}\right\} \operatorname{Pr}\left\{c_{\mathrm{B}, \nu}=1 \mid y_{\mathrm{R}}\right\}\right]$ or the LLR $\lambda_{\mathrm{B}, \nu}=$ $\ln \left(\frac{\operatorname{Pr}\left\{c_{\mathrm{B}, \nu}=0 \mid y_{\mathrm{R}}\right\}}{\operatorname{Pr}\left\{c_{\mathrm{B}, \nu}=1 \mid y_{\mathrm{R}}\right\}}\right)$ is fed to the decoder $\mathrm{B}$ to yield the estimate $\hat{\mathbf{c}}_{\mathrm{B}}$. Finally, the decoder output vectors $\hat{\mathbf{c}}_{\mathrm{A}}$ and $\hat{\mathbf{c}}_{\mathrm{B}}$ are combined to achieve the relay codeword $\mathbf{c}_{R}=\hat{\mathbf{c}}_{\mathrm{A}} \oplus \hat{\mathbf{c}}_{\mathrm{B}}$. Thus, at the relay common network coding is performed as presented in [2] before $\mathbf{x}_{\mathrm{R}}=\mathcal{M}\left\{\mathbf{c}_{\mathrm{R}}\right\}$ is transmitted.

Alternatively, for successive decoding (called S-SCD) the decoding result of the channel with the larger fading gain is subtracted form the receive signal and a common decoding for the second codeword with respect to this interference reduced signal is performed.

\section{B. Joint Channel Decoding and Physical-Layer Network Cod- ing (JCNC)}

For physical-layer network coding the relay is asked to generate from the receive signal $\mathbf{y}_{\mathrm{R}}$ a network coded symbol $\mathbf{x}_{\mathrm{R}}$ being a function of $\mathbf{c}_{\mathrm{A}}$ and $\mathbf{c}_{\mathrm{B}}$ ( or $\mathbf{x}_{\mathrm{A}}$ and $\mathbf{x}_{\mathrm{B}}$ ). To build this relay codeword $\mathbf{c}_{R}$ it is not necessary that the relay knows the source codewords $\mathbf{c}_{\mathrm{A}}$ and $\mathbf{c}_{\mathrm{B}}$ explicitly as observed in [3].

Since $\mathbf{c}_{\mathrm{A}}$ and $\mathbf{c}_{\mathrm{B}}$ are codewords of the same linear channel code $\Gamma$, the modulo- 2 sum $\mathbf{c}_{\mathrm{A} \oplus \mathrm{B}}=\mathbf{c}_{\mathrm{A}} \oplus \mathbf{c}_{\mathrm{B}}$ is also a valid codeword of $\Gamma$. Thus, Joint Channel decoding and physicallayer Network Coding (JCNC) aims to estimate that codeword $\hat{\mathbf{c}}_{\mathrm{A} \oplus \mathrm{B}}$ that caused the observation $\mathbf{y}_{\mathrm{R}}$ most likely using a standard decoding algorithm, i.e., by SPA for LDPC codes. To this end, the APPs for $c_{\mathrm{A} \oplus \mathrm{B}, \nu}=0$ and $c_{\mathrm{A} \oplus \mathrm{B}, \nu}=1$ have to be determined with respect to the corresponding observation $y_{\mathrm{R}}$. The APPs

$$
\operatorname{Pr}\left\{c_{\mathrm{A} \oplus \mathrm{B}, \nu}=\xi \mid y_{\mathrm{R}}\right\}=\sum_{i \in \Psi_{\nu}^{\xi}} P_{i}
$$

can again be calculated using (4). Now, $\Psi_{\nu}^{\xi}$ defines the set of indices, where the $\mathrm{XOR} c_{\mathrm{A} \oplus \mathrm{B}, \nu}=c_{\mathrm{A}, \nu} \oplus c_{\mathrm{B}, \nu}$ is equal to $\xi=\{0,1\}$ according to Tab. I. For example, $\Psi_{1}^{0}=\{0,2,5,7,8,10,13,15\}$ indicates all events where $c_{\mathrm{A} \oplus \mathrm{B}, 1}=0$. For decoding, the APP vector $\left[\operatorname{Pr}\left\{c_{\mathrm{A} \oplus \mathrm{B}, \nu}=\right.\right.$ $\left.\left.0 \mid y_{\mathrm{R}}\right\} \operatorname{Pr}\left\{c_{\mathrm{A} \oplus \mathrm{B}, \nu}=1 \mid y_{\mathrm{R}}\right\}\right]$ or the corresponding LLR

$$
\lambda_{c_{\mathrm{A} \oplus \mathrm{B}, \nu}}=\ln \left(\frac{\operatorname{Pr}\left\{c_{\mathrm{A} \oplus \mathrm{B}, \nu}=0 \mid y_{\mathrm{R}}\right\}}{\operatorname{Pr}\left\{c_{\mathrm{A} \oplus \mathrm{B}, \nu}=1 \mid y_{\mathrm{R}}\right\}}\right)
$$

is fed to the SPA. At the output the estimate for the relay codeword $\mathbf{c}_{\mathrm{R}}=\hat{\mathbf{c}}_{\mathrm{A} \oplus \mathrm{B}}$ is achieved which is then transmitted to both sources after QPSK-modulation.

The basic idea of this approach is the estimation of the XOR of the two source vectors using a common decoder. However, this direct decoding $\mathbf{y}_{\mathrm{R}} \rightarrow \hat{\mathbf{c}}_{\mathrm{A} \oplus \mathrm{B}}$ discards useful information provided by the two channel codes [7]. In order to improve the decoding at the relay, an improved decoding algorithm is presented in the next section.

\section{Generalized Joint Channel Decoding And PhysiCAL-LAYER NETWORK CODING (G-JCNC)}

\section{A. General Approach}

Instead of decoding the source signals separately as presented in Section III-A or by decoding with respect to the XOR as in Section III-B, we propose to decode the two codes jointly within a Generalized Sum-Product Algorithm (G-SPA). Thus, first the decoding $\mathbf{y}_{\mathrm{R}} \rightarrow \hat{\mathbf{c}}_{\mathrm{AB}}$ with respect to $\mathbb{F}_{16}$ is performed and then the physical layer network coding $\hat{\mathbf{c}}_{\mathrm{AB}} \rightarrow \mathbf{c}_{\mathrm{R}}$ is executed by a corresponding mapping rule. This approach fully exploits all available information about the superimposed receive signal as well as the code structure of both channel codes within the decoding.

The basic idea goes back to the Arithmetic-Sum JCNC (AS-JCNC) approach presented in [7]-[9], where the authors restricted the analysis to the transmission of BPSK signals over AWGN channels which results in only three different undisturbed signal levels $\mathcal{S}_{\mathrm{AB}}^{\prime}=\{-2,0,2\}$ where $s_{\mathrm{AB}}^{\prime}=0$ is true for the two cases where $x_{\mathrm{A}} \neq x_{\mathrm{B}}$. However, as the gains $h_{\mathrm{A}}$ and $h_{\mathrm{B}}$ are usually different for fading channels, the knowledge due to the four different receive signal levels should be used in the decoding process by means of a GeneralizedSPA over $\mathbb{F}_{4}$ as presented in [10] and denoted as G-SPA 4 . In this paper, we extend this scheme with respect to the transmission of QPSK signals resulting in a G-SPA over $\mathbb{F}_{16}$ called G-SPA 16 in the sequel.

For the G-SPA 16 scheme a slightly different encoding process compared to the system defined in Sec. II is assumed, where two similar encoders are used to achieve the code bits streams $\mathbf{c}_{\mathrm{A}, 1}=\left[c_{\mathrm{A}, 1}(1) \ldots c_{\mathrm{A}, 1}(L)\right]$ and $\mathbf{c}_{\mathrm{A}, 2}=$ $\left[c_{\mathrm{A}, 2}(1) \ldots c_{\mathrm{A}, 2}(L)\right]$ separately at source $\mathrm{A}$. If $\mathbf{b}_{\mathrm{A}, 1}$ denotes one part of the information sequence at source $A$, the corresponding code word is given by $\mathbf{c}_{\mathrm{A}, 1}=\Gamma\left(\mathbf{b}_{\mathrm{A}, 1}\right)$. Thereby, both code bits affecting the transmit symbol $x_{\mathrm{A}}(\ell)$ have similar connections in the corresponding factor graphs. The encoding at source $\mathrm{B}$ is done likewise.

Basically, each code symbol of a linear channel code consists of the modulo-2 sum of some information bits, e.g., the codebit $c_{\mathrm{A}, \nu}(\ell)$ of $\Gamma$ is given by the sum of the $\nu$-th and the $\mu$-th information bits $c_{\mathrm{A}, 1}(\ell)=b_{\mathrm{A}, 1}(\nu) \oplus b_{\mathrm{A}, 1}(\mu)$. As the same code is used for both code bit streams at both sources, the $\ell$-th 16-ary signal $c_{\mathrm{AB}}(\ell)$ calculates as

$$
\begin{aligned}
c_{\mathrm{AB}}(\ell) & =\left[\begin{array}{l}
c_{\mathrm{A}, 1}(\ell) \\
c_{\mathrm{A}, 2}(\ell) \\
c_{\mathrm{B}, 1}(\ell) \\
c_{\mathrm{B}, 2}(\ell)
\end{array}\right]^{T}=\left[\begin{array}{l}
b_{\mathrm{A}, 1}(\nu) \oplus b_{\mathrm{A}, 1}(\mu) \\
b_{\mathrm{A}, 2}(\nu) \oplus b_{\mathrm{A}, 2}(\mu) \\
b_{\mathrm{B}, 1}(\nu) \oplus b_{\mathrm{B}, 1}(\mu) \\
\left.b_{\mathrm{B}, 2}(\nu) \oplus b_{\mathrm{B}, 2}(\mu)\right]
\end{array}\right] \\
& =b_{\mathrm{AB}}(\nu) \oplus b_{\mathrm{AB}}(\mu) .
\end{aligned}
$$

Thus, $c_{\mathrm{AB}}(\ell)$ is simply given by the sum of the 16-ary information symbols $b_{\mathrm{AB}}(\nu)=b_{\mathrm{A}, 1}(\nu)+b_{\mathrm{A}, 2}(\nu) D+b_{\mathrm{B}, 1}(\nu) D^{2}+$ 
$b_{\mathrm{B}, 2}(\nu) D^{3}$ and $b_{\mathrm{AB}}(\mu)=b_{\mathrm{A}, 1}(\mu)+b_{\mathrm{A}, 2}(\mu) D+b_{\mathrm{B}, 1}(\mu) D^{2}+$ $b_{\mathrm{B}, 2}(\mu) D^{3}$ in $\mathbb{F}_{16}$. Similarly, if a code bit equals the modulo-2 sum of more than two information bits, the symbol $c_{\mathrm{AB}}(\ell)$ is given by the sum of the corresponding 16-ary symbols $b_{\mathrm{AB}}(\cdot)$ in $\mathbb{F}_{16}$. Based on this observation, the overall encoding process of $\mathbf{b}_{\mathrm{AB}} \rightarrow \mathbf{c}_{\mathrm{AB}}$ can be interpreted as a LDPC code over $\mathbb{F}_{16}$ with the restriction, that the elements of the resulting parity check matrix are either 0 or $1+D+D^{2}+D^{3}$. Consequently, a SPA for $\mathbb{F}_{16}$ can be used for decoding [12]. In a similar way also the sum of several code symbols $c_{\mathrm{AB}}(\cdot)$ has to be executed.

\section{B. Messages and Initialization}

The G-SPA 16 determines iteratively the a-posteriori probability of each message symbol $c_{\mathrm{AB}}$ and it is conveniently described over a factor graph which depicts the relations between the variable nodes and the check nodes defined by the parity check matrix $\mathbf{H}$ of the LDPC code [11].

The probability mass function for a 16-ary random variable can be represented by the probability vector $\mathbf{p}=\left[p_{0} \ldots p_{15}\right]$ where $p_{i}$ denotes the probability that the value of the variable is $\mathcal{C}_{\mathrm{AB}}(i)$ with $i \in\{0,1, \ldots, 15\}$ and $\sum_{i=0}^{15} p_{i}=1$ holds. Within the G-SPA 16 these probability vectors are exchanged between the variable nodes and the check nodes as messages. The initial message of variable node $c_{\mathrm{AB}}$ given the received signal $y_{\mathrm{R}}$ equals

$$
\mathbf{p}=\left[\begin{array}{llll}
P_{0} & P_{1} & \ldots & P_{15}
\end{array}\right],
$$

with probabilities $P_{i}=\operatorname{Pr}\left\{c_{\mathrm{AB}}=\mathcal{C}_{\mathrm{AB}}(i) \mid y_{\mathrm{R}}\right\}$ given in (4). Within the G-SPA 16 the same message updating rules at the variable and the check nodes are used as discussed in [11]. Consistently, the update functions at the variable nodes and at the check nodes are defined as VAR and CHK, respectively. Subsequently, the discussion will be restricted to nodes of degree three, i.e., the nodes are connected by three edges. Messages from variable nodes (or check nodes) with degree larger than three can be calculated by

$$
\begin{aligned}
\operatorname{VAR}(\mathbf{p}, \mathbf{q}, \cdots) & =\operatorname{VAR}(\mathbf{p}, \operatorname{VAR}(\mathbf{q}, \operatorname{VAR}(\cdot, \cdot)) \\
\operatorname{CHK}(\mathbf{p}, \mathbf{q}, \cdots) & =\operatorname{CHK}(\mathbf{p}, \operatorname{CHK}(\mathbf{q}, \operatorname{CHK}(\cdot, \cdot)),
\end{aligned}
$$

where $\mathbf{p}$ and $\mathbf{q}$ denote corresponding input messages of the variable nodes (or check nodes) [11].

\section{Output Message of Variable Nodes}

When the two input messages $\mathbf{p}=\left[\begin{array}{lll}p_{0} & \ldots & p_{15}\end{array}\right]$ and $\mathbf{q}=$ $\left[\begin{array}{lll}q_{0} & \ldots & q_{15}\end{array}\right]$ arrive at the variable node $c_{\mathrm{AB}}(\ell)$, the probability that the code symbol $c_{\mathrm{AB}}(\ell)$ is $\mathcal{C}_{\mathrm{AB}}(i), 0 \leq i \leq 15$, is given by

$$
\begin{aligned}
& \operatorname{Pr}\left\{c_{\mathrm{AB}}(\ell)=\mathcal{C}_{\mathrm{AB}}(i) \mid \mathbf{p}, \mathbf{q}\right\} \\
& =\frac{\operatorname{Pr}\left\{\mathbf{p}, \mathbf{q} \mid c_{\mathrm{AB}}(\ell)=\mathcal{C}_{\mathrm{AB}}(i)\right\} \operatorname{Pr}\left\{c_{\mathrm{AB}}(\ell)=\mathcal{C}_{\mathrm{AB}}(i)\right\}}{\operatorname{Pr}\{\mathbf{p}, \mathbf{q}\}} \\
& =\frac{\operatorname{Pr}\left\{c_{\mathrm{AB}}(\ell)=\mathcal{C}_{\mathrm{AB}}(i) \mid \mathbf{p}\right\} \operatorname{Pr}\left\{c_{\mathrm{AB}}(\ell)=\mathcal{C}_{\mathrm{AB}}(i) \mid \mathbf{q}\right\} \operatorname{Pr}\{\mathbf{p}\} \operatorname{Pr}\{\mathbf{q}\}}{\operatorname{Pr}\left\{c_{\mathrm{AB}}(\ell)=\mathcal{C}_{\mathrm{AB}}(i)\right\} \operatorname{Pr}\{\mathbf{p}, \mathbf{q}\}} \\
& =\beta p_{i} q_{i},
\end{aligned}
$$

where $\beta=\frac{\operatorname{Pr}\{\mathbf{p}\} \operatorname{Pr}\{\mathbf{q}\}}{\operatorname{Pr}\left\{c_{\mathrm{AB}}(\ell)=\mathcal{C}_{\mathrm{AB}}(i)\right\} \operatorname{Pr}\{\mathbf{p}, \mathbf{q}\}}$ is a normalization factor. Since the sum of the probabilities (12) should be 1 over all $i$, the normalization factor equals $\beta=1 / \sum_{i=0}^{15} p_{i}$. Thus, the output message of the variable node is

$$
\operatorname{VAR}(\mathbf{p}, \mathbf{q})=\beta\left[\begin{array}{llll}
p_{0} q_{0} & p_{1} q_{1} & \cdots & p_{15} q_{15}
\end{array}\right] .
$$

\section{Output Message of Check Nodes}

A specific parity check equation is satisfied, if the $\mathbb{F}_{16}$ sum of the corresponding 16-ary symbols equals zero, i.e., $c_{\mathrm{AB}}(\nu) \oplus c_{\mathrm{AB}}(\mu) \oplus c_{\mathrm{AB}}(\ell)=0$. Assume the two input message vectors from the variable nodes $c_{\mathrm{AB}}(\nu)$ and $c_{\mathrm{AB}}(\mu)$ are $\mathbf{p}=\left[\begin{array}{lll}p_{0} & \ldots & p_{15}\end{array}\right]$ and $\mathbf{q}=\left[\begin{array}{lll}q_{0} & \ldots & q_{15}\end{array}\right]$, respectively. The probability that the parity check equation is satisfied under the assumption that $c_{\mathrm{AB}}(\ell)$ is fixed to $\mathcal{C}_{\mathrm{AB}}(i)$ equals

$$
\begin{aligned}
& \operatorname{Pr}\left\{c_{\mathrm{AB}}(\ell)=\mathcal{C}_{\mathrm{AB}}(k) \mid \mathbf{p}, \mathbf{q}\right\} \\
& =\sum_{i=0}^{15} \operatorname{Pr}\left\{c_{\mathrm{AB}}(\nu)=\mathcal{C}_{\mathrm{AB}}(i), c_{\mathrm{AB}}(\mu)=\mathcal{C}_{\mathrm{AB}}\left(\psi_{k, i}\right) \mid \mathbf{p}, \mathbf{q}\right\} \\
& =\sum_{i=0}^{15} p_{i} q_{\psi_{k, i}} .
\end{aligned}
$$

Here, the variable $\psi_{k, i}$ denotes the integer representation of $\mathcal{C}_{\mathrm{AB}}(k) \oplus \mathcal{C}_{\mathrm{AB}}(i)$, e.g., $\psi_{1,3}=2$ as $\mathcal{C}_{\mathrm{AB}}(1) \oplus \mathcal{C}_{\mathrm{AB}}(3)=1 \oplus$ $(1+D)=D=\mathcal{C}_{\mathrm{AB}}(2)$. Finally, the message vector out of one check node equals

$$
\operatorname{CHK}(\mathbf{p}, \mathbf{q})=\left[\begin{array}{c}
\operatorname{Pr}\left\{c_{\mathrm{AB}}(\ell)=0 \mid \mathbf{p}, \mathbf{q}\right\} \\
\vdots \\
\operatorname{Pr}\left\{c_{\mathrm{AB}}(\ell)=1+D+D^{2}+D^{3} \mid \mathbf{p}, \mathbf{q}\right\}
\end{array}\right]^{T}
$$

\section{E. Finalization and PLNC Mapping}

The decoding is stopped if all parity check equations are fulfilled or the maximum number of iterations is reached. Otherwise, the algorithm proceeds with steps $C$ and $D$ for further iterations until one of these conditions is fulfilled. At the end the decoding algorithm generates the APP vector $\mathbf{p}$ with $p_{i}=\operatorname{Pr}\left\{c_{\mathrm{AB}}(\ell)=\mathcal{C}_{\mathrm{AB}}(i) \mid \mathbf{y}_{\mathrm{R}}\right\}$ for each code symbol $c_{\mathrm{AB}}(\ell)$ and the PLNC mapping is done by

$$
c_{\mathrm{R}}(\ell)=\left\{\begin{array}{ll}
00 & \text { if } \underset{i}{\operatorname{argmax}} p_{i}=\{0,5,10,15\} \\
10 & \text { if } \underset{i}{\operatorname{argmax}} p_{i}=\{1,4,11,14\} \\
01 & \text { if } \underset{i}{\operatorname{argmax}} p_{i}=\{2,7,8,13\} \\
11 & \text { if } \underset{i}{\operatorname{argmax}} p_{i}=\{3,6,9,12\}
\end{array} .\right.
$$

If, e.g., $p_{13}$ is the maximum value over all $p_{i}$ for $0 \leq i \leq$ 15, the 4-tuple $c_{\mathrm{AB}}=\left[\begin{array}{llll}1 & 0 & 1 & 1\end{array}\right]$ was transmitted most likely. Thus, the XOR of the corresponding source code words equals $c_{\mathrm{A}} \oplus c_{\mathrm{B}}=\left[\begin{array}{ll}0 & 1]\end{array}\right]$ leading to the relay codeword $c_{\mathrm{R}}=\left[\begin{array}{ll}0 & 1\end{array}\right]$.

\section{Simulation Results}

In this section, the performance of the proposed G-JCNC scheme using G-SPA 16 is compared to the separated channel decoding schemes P-SCD and S-SCD of Sec. III-A, and the JCNC of Sec. III-B. Optimized LDPC codes for code rate $R_{c}=0.4$ and codeword length $N=1000$ for the schemes of Sec. III and $N=500$ for G-JCNC are used to yield equal 
frame length of $L=500$ [13]. All SPA decoders perform 10 iterations. For the first simulations, a normalized fading channel is considered, where the channel for $\mathrm{A}$ is always $h_{\mathrm{A}}=1$ and the channel for $\mathrm{B}$ is uniformly distributed on the unit circle with an angle between $\pi / 8$ and $3 \pi / 8$, i.e., $h_{\mathrm{B}}=\exp (j \phi)$ with $\phi \sim U(\pi / 8,3 \pi / 8)$, but remains constant for one transmission block. Thus, both channels have the same reliability, but a strong overlapping of the QPSK symbols of $\mathrm{A}$ and $\mathrm{B}$ is avoided by the restricted phase rotation. This enables an investigation of the decoding gain of the different approaches neglecting the influence of fading or diversity.

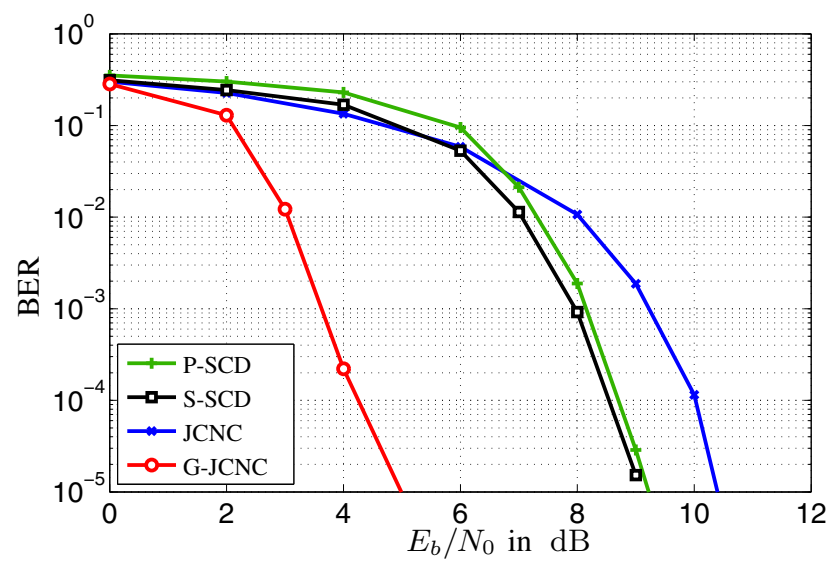

Fig. 2. BER for parallel and successive separated channel decoding ( $\mathrm{P}$ SCD and S-SCD), joint channel decoding and physical-layer network coding (JCNC), and generalized JCNC (G-JCNC).

Fig. 2 shows the end-to-end bit error rate (BER) averaged over both sources $\mathrm{A}$ and $\mathrm{B}$ for varying $E_{b} / N_{0}$. The JCNC approach is not able to estimate the relay codeword sufficiently leading to degraded performance. In contrast, the separated decoding approaches P-SCD and S-SCD offer improved performances. However, the new approach G-JCNC significantly outperforms all other schemes under investigation. In comparison to the SSCD scheme a gain of approximately $4 \mathrm{~dB}$ for BER of $10^{-5}$ is achieved. This justifies the application of a G-SPA over $\mathbb{F}_{16}$. If the channel $h_{\mathrm{B}}$ contains arbitrary elements on the unit circle, it is likely that receive signal space $\mathcal{S}_{\mathrm{AB}}$ contains only four well distinguishable signals. Thus, the performance of all detection schemes would be bad.

Fig. 3 shows BERs for an OFDM system where 500 out of $N_{C}=512$ sub carriers are used for data transmission. The fading coefficients $h_{\mathrm{A}}$ and $h_{\mathrm{B}}$ are given by the IFFT of the corresponding frequency-selective fading channels of order 7 containing i.i.d. elements. Thus, on some sub carriers the receive signal space does not contain 16 well distinguishable points leading to similar APPs for different events $\mathcal{S}_{\mathrm{AB}}(i)$. Consequently, the gains for our new approach are reduced in comparison to Fig. 2. However, improvements of more than $1 \mathrm{~dB}$ for BER of $10^{-5}$ are still achieved.

\section{SUMMARY}

In this paper joint channel decoding and physical-layer network coding in two-way relay systems with QPSK transmission is investigated. The new decoding approach Generalized

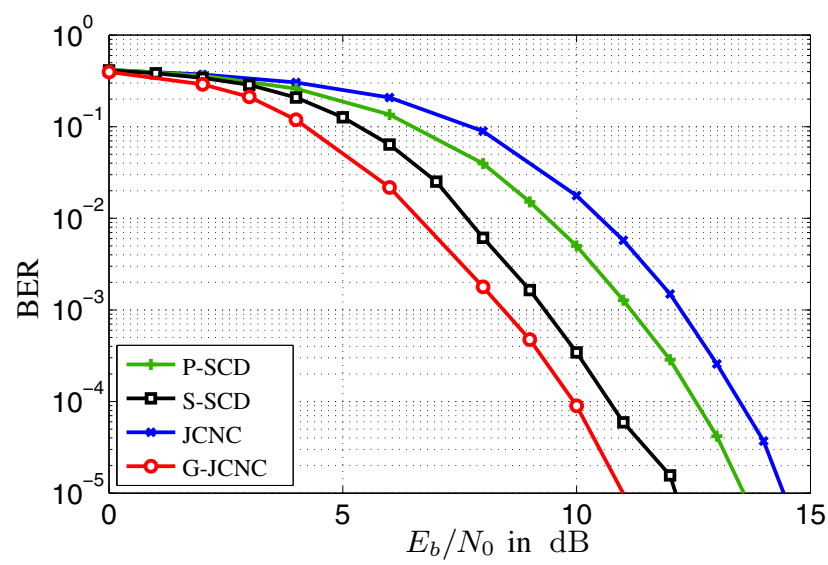

Fig. 3. BERs for an OFDM two-way relay system with 500 out of $N_{C}=512$ sub carriers used for data transmission, channel order 7 .

Joint Channel decoding and physical-layer Network Coding (G-JCNC) was presented to estimate the XOR of the two source codewords at the relay from the superimposed receive signal exploiting the overall channel code. To this end a Generalized Sum-Product Algorithm (G-SPA) was derived which performs decoding with respect to $\mathbb{F}_{16}$. The simulation results show a significant performance improvement in comparison to the schemes from the literature.

\section{REFERENCES}

[1] R. Ahlswede, N. Cai, S.-Y.R. Li, and R.W. Yeung, "Network Information Flow," IEEE Trans. on Information Theory, vol. 46, no. 4, pp. 12041216, July 2000.

[2] C. Fragouli, J.Y. Boudec, and J. Widmer, "Network Coding: An Instant Primer," ACM SIGCOMM Computer Communication Review, vol. 36, no. 1, pp. 63-68, Mar. 2006.

[3] S. Zhang, S.C. Liew, and P. Lam, "Hot Topic: Physical Layer Network Coding," in Proc. International Conference on Mobile Computing and Networking (MobiCom), Los Angeles, CA, USA, 2006, pp. 358-365.

[4] P. Popovski and H. Yomo, "The AntiPackets Can Increase the Achievable Throughput of a Wireless MultiHop Network," in IEEE Proc. Int. Conference on Communications (ICC), Istanbul, Turkey, June 2006.

[5] S. Zhang, S.C. Liew, and P.P. Lam, "Physical Layer Network Coding," "http://arxiv.org/abs/0704.2475".

[6] A. Zhan and C. He, "Joint Design of Channel Coding and Physical Network Coding for Wireless Network," in IEEE Proc. Int. Conference on Neural Networks and Signal Processing, Zhejiang, China, June 2008.

[7] S. Zhang and S.C. Liew, "Channel Coding and Decoding in a Relay System Operated with Physical-Layer Network Coding," IEEE Journal on Selected Areas in Comm., vol. 27, no. 5, pp. 788-796, Oct. 2009.

[8] Y. Lang, D. Wübben, and K.D. Kammeyer, "An Improved Physical Layer Network Coding Scheme for Two-Way Rrelay Systems," in Proc. International ITG Workshop on Smart Antennas (WSA), Bremen, Germany, Feb. 2010.

[9] Y. Lang and D. Wübben, "Generalized Joint Channel Coding and Physical Network Coding for Two-way Relay Systems," in IEEE Proc. Vehicular Technology Conference (VTC), Taipei, Taiwan, May 2010.

[10] D. Wübben and Y. Lang, "Generalized Sum-Product Algorithm for Joint Channel Decoding and Physical-Layer Network Coding in Two-Way Relay Systems," in IEEE Proc. Global Communications Conference (GLOBECOM), Miami, FL, USA, Nov. 2010.

[11] F.R. Kschischang, B.J. Frey, and H.A. Loeliger, "Factor Graphs and the Sum-Product Algorithm," IEEE Trans. on Information Theory, vol. 47, no. 2, pp. 498-519, Feb. 2001.

[12] M.C. Davey and D. MacKay, "Low Density Parity Check Codes over GF $(q)$," IEEE Commun. Letters, vol. 2, no. 6, pp. 165-167, June 1998.

[13] R. Urbanke, "LDPC: Optimization of LDPC Codes for BP Decoder," online: http://ipgdemos.epfl.ch/ldpcopt/. 